\title{
HIPÓTESIS JACOBIANA. UN EJERCICIO DE GIMNASIA MENTAL PARA ECONOMISTAS INSPIRADO EN LAS ENSEÑANZAS DEL DR. BARBA-ROMERO CASILLAS
}

\section{JACOBIAN HYPOTHESIS. AN EXERCISE IN MENTAL GYMNASTICS TO ECONOMISTS INSPIRED BY THE TEACHINGS OF DR. BARBA-ROMERO CASILLAS}

Carlos Díaz Gómez: Universidad de Alcalá de Henares. Madrid (España) carlos_diazgomez@yahoo.com

\section{CURRÍCULUM VITAE DE CADA AUTOR}

Doctor en Filosofía por la Universidad Complutense de Madrid (España) con Premio Extraordinario, Licenciado en Derecho por la Universidad Complutense de Madrid (España) con Premio Extraordinario, Licenciado en Altos Estudios Constitucionales con Premio Extraordinario y Profesor de la Universidad Complutense de Madrid (España). Autor de numerosos libros y artículos en revistas especializadas y universitarias.

\section{RESUMEN}

Se trata de, mediante un ejemplo, diferenciar entre ecuación, o lo que es lo mismo afirmación matemática que se satisface, cuando tiene solución, para cierto o ciertos valores, que pueden ser infinitos o ninguno, e identidad, o lo que es lo mismo objeto matemático de apariencia similar, casi idéntica, y sin embargo, de naturaleza o 
propiedades muy distintas. Para ello se usa una hipótesis jacobiana y las diferencias no son muchas.

\section{PALABRAS CLAVE}

Ecuación - Identidad - Hipótesis jacobiana

\section{ABSTRACT}

It is through an example, the difference between equation, or what is the same mathematical statement is satisfied, when you have solution for certain or certain values, which can be infinite if any, and identity, or what is the same mathematical object of similar appearance, almost identical, and yet in nature or very different properties. This hypothesis using a Jacobian and the differences are not great.

\section{KEY WORDS}

Equation - Identity - Hypothesis Jacobian

\section{TEXTO:}

Sea

$\mathrm{F}(\mathrm{x} 1, \mathrm{x} 2)=1=x 1 \times 2$

$x 1 \times 2=1$ 
$x 1=1 / x 2$

"Empecemos con un sistema dinámico (NOTA 1) tan sencillo que casi da vergüenza llamarle sistema".

No sin antes referirnos a "Why Johnny Hates Math", pequeño papel escrito en 1956 por Karl Menger, y que podrán encontrar en Selected papers in logic and foundations, didactics, economics, en nuestra Biblioteca de la Facultad de Ciencias Económicas y Empresariales de la Universidad de Alcalá, antigua y primera Universidad Complutense.

$J(d F / d x 1, d F / d x 2)=(x 2, x 1)$

Una vez definida y calculada la matriz jacobiana, la evalúo en un punto (NOTA2) que satisfaga el sistema, esto es, un punto que satisfaga ambas (NOTA3) ecuaciones que no identidades- simultáneamente. (Aunque parezca la misma ecuación escrita de dos formas distintas y no un sistema de dos ecuaciones, vea lo que sucede cuando x2 vale 0$)$.

Me importa señalar una noción fundamental en matemáticas y que en economía ha de estar siempre nítida, so pena de perecer, no sólo en nuestros exámenes, sino también en nuestras disquisiciones teóricas posteriores. La aclaración y el excelente ejemplo se los debo, para mi fortuna, al libro del profesor E. Costa Matemáticas para economistas. Se trata de la distinción entre ecuación -afirmación matemática que se satisface, cuando tiene solución, para cierto o ciertos valores, que pueden ser infinitos (NOTA4) o ninguno (NOTA5)- e identidad -objeto matemático de apariencia similar, casi idéntica, y sin embargo, de naturaleza o propiedades muy distintas-. Se diferencian en poco -alguien podría decir-, mas aquello que las distingue es de enorme trascendencia, y por tanto, lo poco es aquí, como en casi todo, mucho. Del 
mismo modo, alguien podría decir que entre acertar y fallar cuando se lanza una bala no hay tanta diferencia, si se considera que en ambas acciones se dispara la pistola y sale despedido el proyectil. Sin embargo, si nuestra vida depende del acierto, la diferencia, que desde un cierto punto de vista es pequeña, e incluso mínima, lo es todo. Un ejemplo similar es de aplicación a las situaciones "casi quedarse embarazada", "casi perder un millón de dólares" o "casi ser arrollado por un autobús cuando se camina distraído por la gran ciudad". Nótese, por tanto, que lo pequeño o marginal puede ser decisivo en las cosas humanas, y así en las económicas. Puesto que la dimensión económica, como la dimensión filosófica o la jurídica, nos pertenece con independencia de nuestra voluntad. $\mathrm{Y}$ es, por tanto, no ya importante, sino imprescindible dedicarles nuestro tiempo y nuestra atención.

Pues bien,

$\mathrm{Y}=\mathrm{DA}(\mathrm{NOTA})$

$\mathrm{y}$

$(a+b) 2=a 2+2 a b+b 2$

son identidades (aunque sus miembros estén separados por símbolos diferentes, un guiño del profesor): se satisfacen para cualquier valor real elegido para las letras Y ó DA, a y b. No sucede lo mismo con la expresión

$a+b=7$,

que tiene infinitas -ipero no todas!- soluciones. El ejemplo gana en "luminosidad" cuando nos detenemos en la ecuación

$\mathrm{a}+2=7$ 
que tiene solución única: sólo puede ser verdad cuando $a=5$. Ningún otro valor para la incógnita a podría nunca satisfacer la ecuación.

Tal es la naturaleza de este objeto matemático que llamamos "ecuación"; es como una cerradura que sólo pudiera girar con una llave concreta, cierta y determinada, o bien con cualquier elemento de un conjunto de llaves que compartan todas ellas una determinada cualidad numérica. Es verdad que la ecuación es sólo una representación teórica, como el triángulo isósceles en trigonometría o el plano inclinado de los físicos; y no tiene corazón, ni modifica sus cualidades con el paso de los siglos. Pero sólo puede vivir en el mundo teórico al que pertenece.

Ahora, retomando nuestro tema, calculo el valor de la matriz jacobiana evaluada en cada uno de los cuatro puntos que he elegido. En realidad, me basta con uno. Lo que quiero averiguar es "si el jacobiano es de rango completo m", cuál es el orden del determinante de la matriz jacobiana del sistema evaluada en algún punto que lo satisfaga. Nótese bien que, antes de saber si puedo reescribir sin modificar un ápice su significado - para lo cual ha de verificarse la denominada hipótesis jacobiana

$x 1 \times 2=1 \operatorname{con} x 1=1 / x 2$,

una operación aparentemente tan simple, inocente y rutinaria-, he restringido mi conjunto de números reales representados por $x 1$ y $x 2$ a aquellos tales que su producto vale 1 (no pierda de vista que el cero está excluido). Esto quiere decir que, limitándonos a ese subconjunto de pares de números reales $(x 1, x 2)$ tales que $x 1 \times 2=$ 1 , la ecuación se convierte en $\times 1 \times 2=1$. Si alguien nos dijese ahora «tengo aquí x1 = 1 y $x 2=6$, ¿qué obtengo al introducirlos en tu función?», le tendríamos que decir que no sabemos (NOTA7). Lo mismo sucedería si alguien se presentase con un par de números que fuesen irracionales, o con uno nada más (NOTA8), con 271/2, por ejemplo, o con p, iqué decir de (-6)1/2! Evidentemente, desde el punto de vista 
puramente matemático, si admitimos las soluciones del sistema en el cuerpo de los números reales (o de los complejos para el último caso), no hay nada que decir, pero podemos restringir las soluciones al subconjunto de los números racionales. ¡Se trata de una limitación más que superlativa! Lo digo porque es típico de un cierto tipo de "economistas" derivar, de tan reducidísimos casos particulares, previamente preparados y acordes con su sentir, prescripciones universales de política económica que a todos nos afectan -muchas veces de modo fatal, y casi diríase ineluctable-, y lo presentan como algo, no ya necesario, sino imprescindible, muy bien elaborado y muy elevado, que sólo se deja conocer por sus privilegiadas cabezas, cuando es sólo producto de la estrechez de sus planteamientos, de la reducidísima e irreal colección de sus supuestos de partida. Es típico del profesor de teoría económica dirigirse al auditorio y decir -antes de llenar diez o doce pizarras sin demasiado rigor, y fingiendo que cree hacerse pasar por alguien que sabe algo fundamental que los demás ignoramos- que va a hacer dos o tres simplificaciones muy concretas que sabe que no son verdad (habitualmente son tres despropósitos vitales que brotan naturalmente de su sentir de buen burgués, de su posmodernidad manifiesta, diría quizá McCloskey), pero que son necesarias para poder avanzar. Después aburre miserablemente al auditorio que, a veces, ni finge seguirle, y acaba concluyendo -ya ha perdido la humildad inicial de sus "supuestos" y se torna soberbio de mala ley- en qué dirección concreta ha de orientarse la política económica del futuro.

Como el jacobiano tiene rango completo, se cumple la hipótesis jacobiana. Luego, tanto $x 1$ como $x 2$ son -o pueden ser- variables endógenas. Ahora bien, si queremos resolver, estamos en la obligación ineludible de fijar, en primer lugar, un valor, bien para $x 1$, bien para x2. La elección de la variable fijada convierte a ésta, automáticamente, en variable exógena del modelo (NOTA9), en el caballo que pongo delante de mi carreta. Luego, determino su valor arbitrariamente, porque sí, porque me apetece. Es un valor que me saco convenientemente de la manga y coloco sobre la 
mesa a la vista de todos, como si fuese un mago profesional con su reloj rectangular de números romanos y su paquete de americanos.

\section{El público no dice nada.}

Parece que da igual, que no tiene mayores repercusiones ni significado elegir, como variable exógena o predeterminada, x1 ó x2; que me da igual a que b; 4 que 40. Pues no. Es tan distinto como poner la carreta delante o detrás de los bueyes (NOTA10) poner el carro delante del caballo puede ser fatal, tanto como tomar el rábano por las hojas-. ¿Cuando llueve, la gente saca el paraguas o, cuando la gente saca el paraguas, llueve? Este es un ejemplo que el profesor Arellano nos dijo ponía Hicks al tratar la dependencia estadística y la causalidad, o la correlación y la dependencia. Recuerde que "correlación no implica dependencia, pero dependencia sí implica correlación". Ahora bien, recuerde también que la correlación que mide el famoso R2 es de tipo lineal, como la autocorrelación que detecta el test de Durbin-Watson. Y no de otro tipo. Así, dos variables, convenientemente aisladas del resto del mundo material e inmaterial, pueden presentar una perfecta correlación cuadrática, como x e y en y = x2, y, sin embargo, su R2 será estadísticamente igual a cero (NOTA11), y los contrastes estadísticos para detectar la multicolinealidad fundamentados en el coeficiente de correlación de Pearson, r, que hasta donde yo sé son todos, tendrán un error de tipo I de tamaño colosal. Pues bien, en casos tales como el de la lluvia y el paraguas, que son los propios del mundo económico, no es en absoluto igual decir x1 $=1 / x 2$ que $x 2=1 / x 1$. No nos hallamos en el mundo propio de la mecánica clásica, en el cual hemos definido un sistema inercial, un punto fijo y perfectamente inmóvil e invariable -como el "stock" áureo del planeta, que apenas cambia con el paso de los siglos- al cual referirlo todo. Nadie sabe muy bien qué pueda ser eso que llamamos la cantidad nominal de dinero. Por eso, la definimos primero. Al hacerlo, todo el mundo rehace sus carteras y altera sus posiciones, y no se sabe muy bien si lo hacen porque ha cambiado la definición legal de dinero o por razones que nada tienen que 
ver con tal modificación, o por una "mezcla" harto mal conocida y peor formulada de ambas razones. Y los llamados economistas vuelven a escribir otra ecuación y a tratar de estimar sus parámetros, que "se mueven más que los precios", a la manera de decir de "Chiquito de La Calzada" (NOTA12). ¡Porqué son variables aleatorias! gritará quizá alguien. Ésta es la ley de Goodhart, según la cual «sea cual sea el agregado monetario que se elija como variable objetivo, quedará distorsionado por el sólo hecho de haber sido elegido.»

«Puesto que las leyes empíricas de la física -nos dice la Enciclopedia Británica- no dependen de una especial o accidental elección de marcos de referencia seleccionados para representar relaciones físicas y configuraciones geométricas, el análisis vectorial constituye una herramienta ideal para el estudio del universo físico. La introducción de un marco de referencia especial o sistema de coordenadas establece una correspondencia entre vectores y conjuntos de números que representan componentes de vectores en ese marco, y permite inducir leyes concretas para operar con esos conjuntos de números, leyes que se derivan de las que rigen para operar con segmentos de recta».

En el mundo de Euclides todo encaja a la perfección. No hay curvas. O, más propiamente, todas las curvas son rectas, como en Castilla. Por eso llega a resultados tan infalibles e impepinables. Sus hipótesis de partida se lo permiten.

Sin embargo, cuando abandonamos el mundo propio del álgebra lineal y de las variables independientes $\mathrm{u}$ ortogonales $\mathrm{y}$ decidimos introducir una ecuación aparentemente muy simple -y que no lo es tanto, pues ya no es lineal- como x1 x2 = 1, el mundo incontestable y determinista de las matemáticas elementales se aleja de nosotros a velocidad de crucero, y nuestro nuevo puerto se ha convertido en un lugar ajeno y extraño, en el que nuestras viejas certezas han perdido su validez y su vigencia. Y el suelo se mueve al andar; y nuestra estructura ósea se transforma en 
una especie de pegamento chicloide siempre renuente a la petrificación propia del mundo clásico. ¿Qué hacer? ¿Mirar hacia atrás y tener fe en lo desconocido y aprender a vivir deportivamente -abandonando el concepto burgués de la existencia-, a disfrutar, a no lamentarnos mientras nos internamos por el lado difícil y problemático de las cosas, que será con seguridad al final la forma más cómoda y económica de vivir? Creo que Quevedo -el retorcido cojo, como le llamaba Ortegapensaría así.

Supongamos entonces que Quevedo es un economista y que recurrimos a él para predecir la evolución del tipo de cambio euro/dólar. ¿Es esto intolerable o acertado? Es pasar ya de castaño oscuro. «Se pierde usted -mi profesor parece haberse enfadado conmigo-, lo mezcla todo; es usted anárquico, tanto en sus planteamientos como en su ejecución. Parece usted español. Vuelva inmediatamente al mundo ordenado, aunque no muy preciso, de los diagramas de fases, en el que, si bien no todo es del todo predecible, hay poco sitio - ¿poco sitio?- para el azar y la sorpresa; a lo mejor las dos categorías de mayor impacto director en el devenir económico de pueblos, personas y culturas, y hasta enteras civilizaciones (NOTA 13). Cíñase usted al programa, al triste y aburrido programa aprobado por el Ministerio de Educación, y prostitúyase con naturalidad pasmosa, como si fuese un profesor de universidad.»

\section{Continuará...}

\section{$\underline{\text { Notas }}$}

NOTA 1: Lo estático es un caso particular de lo dinámico, y no al revés (véase Schumpeter, 1954 ["History of Economic Analysis", twelfth impression, 1981], p. 964 [Traduc. castellana, 1971, p. 1050]).

Volver al párrafo Volver al principio del artículo Volver al principio 
NOTA 2: He elegido los puntos A, B, C y D del tipo $\mathrm{P}=(\mathrm{x} 1, \mathrm{x} 2)$.

Así, $\mathrm{A}=(1 / 2,2) ; \mathrm{B}=(1 / 4,4) ; \mathrm{C}=(5,1 / 5)$ y $\mathrm{D}=(1,1)$.

Volver al párrafo Volver al principio del artículo Volver al principio

NOTA 3: Si un punto satisface sólo una de las ecuaciones, el sistema no tendría solución: nos hallaríamos ante dos afirmaciones contradictorias o incompatibles, ante dos sistemas de una sola ecuación, pues no hay que olvidar que una ecuación es un caso particular de sistema: aquél en el que $\mathrm{m}=1$, siendo $\mathrm{m}$ el número de ecuaciones que lo conforman ( $\mathrm{n}$ representa su número de incógnitas o variables; $\mathrm{n}=2$ en nuestro ejemplo).

Volver al párrafo $\quad$ Volver al principio del artículo $\quad$ Volver al principio

NOTA 4: Si la solución no es única (teorema fundamental del álgebra para sistemas lineales) el sistema tiene infinitas soluciones, o dicho de otro modo, si hay 2 soluciones, hay también infinitas soluciones. El teorema fundamental del álgebra asegura que cualquier sistema lineal con tantas ecuaciones como incógnitas $(m=n)$, si tiene solución, es única, a menos que alguna de las ecuaciones dependa linealmente de otra u otras de las ecuaciones del sistema. Esto sucede en los sistemas lineales, pero, iy en los no lineales (por ejemplo: $x+y=7 ; x y=5)$ ?; iy en los sistemas de ED (ecuaciones en diferencias) y de Ed (ecuaciones diferenciales) -los sistemas propiamente dinámicos-? Según explican De Grauwe, Dewachter y Embrechts, en la página 3 de su libro de 1993 Exchange Rate Theory, los sistemas de ecuaciones diferenciales lineales -supongo que homogéneas, porque, en caso contrario, creo que nos quedamos sin teoría, aunque haya gente tan brillante en el MIT- obedecen el principio de superposición, de modo que si A y B son soluciones de una Ed, entonces A + B también lo es. ¿Y qué hay de

$2 \mathrm{~A}+\mathrm{B}$ ó de $\mathrm{k} \mathrm{A}+\mathrm{B}$, 
para todo número real k? La respuesta es "sí" si se trata de una EdLH (Ecuación diferencial lineal homogénea). Si la ecuación es no homogénea, A podría ser solución de la ecuación sin termino independiente (parte homogénea) y B una solución de la ecuación completa (no homogénea):

k A + B sería entonces solución de la ecuación completa (no homogénea).

Luego, si la solución de la ecuación algebraica no es única, el sistema tiene infinitas soluciones, sea cual fuere el tipo de sistema, siempre que sea lineal.

Volver al párrafo Volver al principio del artículo Volver al principio

NOTA 5: $\ln -2=x$ es una ecuación sin solución; $0 x=23$, otra (un poco artificiosa, pero eso son las matemáticas, puro artificio; otra cosa es que nos puedan llevar muy lejos, que sean utilísimas y hasta imprescindibles).

Volver al párrafo Volver al principio del artículo $\quad$ Volver al principio

NOTA 6: Esta identidad, en la que Y representa el PIB y DA la "demanda agregada", esconde detrás una teoría revolucionaria debida a John Maynard Keynes. Pura retórica sin mayor valor científico para unos, y santo sacramento de una nueva fe, para otros; es tan famosa en economía como en física la ecuación -que no identidad$\mathrm{E}=\mathrm{mc}$.

Volver al párrafo $\quad$ Volver al principio del artículo $\quad$ Volver al principio

NOTA 7: Le diríamos que estamos en otra curva de nivel. No sea usted tan listo. Volver al párrafo Volver al principio del artículo Volver al principio

NOTA 8: Si se trata sólo de uno de los dos números o factores, basta con multiplicar por su inverso para conseguir un 1. Esto es obvio, como es obvia la distinción entre 
un número real y un número racional. Todo es obvio cuando se sabe y un misterio cuando se ignora o no se recuerda bien. Por eso, éste es un trabajo plagado de notas. Todo número racional es real, pero no todo número real es racional. No puede decirse lo mismo de las personas, de donde se deduce que las personas no son números. Es ésta una deducción tan elemental, que uno duda de que sea una verdadera deducción y no una repetición sin más, una tautología o una perogrullada. Pero la gimnasia es así. Hay que calentar un poco.

Volver al párrafo Volver al principio del artículo $\quad$ Volver al principio

NOTA 9: Habitualmente un modelo económico es algo más que una sola ecuación. Suele ser una colección de metáforas y un cubilete con dos dados. Aunque es más normal que se componga de:

-una identidad contable,

-una ecuación de comportamiento,

-dos variables de ajuste.

Volver al párrafo Volver al principio del artículo Volver al principio

NOTA 10: Mettre la charrue avant les bœufs. To put the cart before the horse .

Volver al párrafo Volver al principio del artículo Volver al principio

NOTA 11: Lo he leído en el libro de Johnston \& DiNardo Econometric Methods (1997).

Volver al párrafo $\quad$ Volver al principio del artículo $\quad$ Volver al principio

NOTA 12: Memorable humorista español que saltó a la fama en la segunda mitad de la década de los 90 y a quien la gente profesa gran cariño y reconocimiento.

Volver al párrafo Volver al principio del artículo Volver al principio 
NOTA 13: Y aquí llegamos al gran debate de la ciencia macroeconómica actual. ¿Es realmente una variable económica función del tiempo? ¿O somos nosotros los que, al suponerlo así, se lo "exigimos" para poder aplicar nuestra limitada y primitiva teoría de ecuaciones diferenciales, olvidándonos luego de que hemos sido nosotros, por nuestra imposibilidad o incapacidad de hacer algo mejor, quienes hemos introducido esa limitación? 